\title{
An Investigation of Functional and Anatomical Connectivity Using Magnetic Resonance Imaging
}

\author{
Martin A. Koch, ${ }^{1}$ David G. Norris, ${ }^{2}$ and Margret Hund-Georgiadis \\ Max Planck Institute of Cognitive Neuroscience, Stephanstraße 1a, D-04103 Leipzig, Germany
}

Received May 1, 2001

This article examines functional and anatomical connectivity in healthy human subjects measured with magnetic resonance imaging methods. Anatomical connectivity in white matter is obtained from measurements of the diffusion tensor. A Monte-Carlo simulation determines the probability that a particle diffuses between two points, with the probability of a jump in a particular direction from a given voxel being based on the local value of the diffusion tensor components. $F$ unctional connectivity between grey matter pixels is assessed without recourse to a specific activation paradigm, by calculating the correlation coefficient between random fluctuations in the blood oxygenation level-dependent signal time course in different pixels. The methods are used to examine the anatomical and functional connectivities between crowns of adjacent gyri. A high functional connectivity was found between grey matter pixels, with white matter displaying only very low correlation. A comparison of the measurements of anatomical and functional connectivity found that there is no simple correlation between these measures, except that low values of functional connectivity were not found together with high values of anatomical connectivity. Furthermore pairs of regions situated around the central sulcus indicated a dependence of the two connectivity measures on each other. These results are in accordance with an interpretation that regions which are clearly directly linked by white matter fiber tracts should show high functional connectivity, but that the inverse need not be true as functional connectivity may also be indirectly mediated via more distant grey matter regions. 2002 Elsevier Science (USA)

\footnotetext{
${ }^{1}$ To whom correspondence and reprint requests should be addressed at present address: Universitätsklinikum Hamburg-E ppendorf, Neurologische Klinik, Martinistraße 52, D-20246 Hamburg, Germany. Fax: +49-40-42803-5086. E-mail: mkoch@uke.unihamburg.de.

${ }^{2}$ Present address: FC Donders Centre for Cognitive Neuroimaging, Trigon 181, P.O. Box 9101, NL-6500 HB Nijmegen, The Netherlands.
}

Key Words: connectivity; functional MRI; diffusion tensor imaging; fiber tracking.

\section{INTRODUCTION}

It is a fundamental aim in cognitive neuroscience to discover anatomical features that reflect the functional organization of the brain. In this paper both functional and anatomical connectivity in the human brain are examined using Magnetic Resonance Imaging (MRI) techniques and the results of the investigations are compared. Specifically, anatomical connectivity is assessed using parameters obtained from the diffusion tensor of water in anisotropic white matter, and functional connectivity is examined by measuring the correlation in spontaneous fluctuations of the Blood Oxygenation Level-Dependent (BOLD) (Ogawa et al., 1990a,b) signal between voxels (Biswal et al., 1995).

The rationale for this choice of methods is as follows: diffusion tensor imaging (DTI) (Basser et al., 1994a,b) is unique in its ability to obtain information concerning the orientation of white matter fiber tracts with cross sections of at least several imaging voxels in size. Using this method it has proven possible to track fiber pathways over extended regions of the brain, producing results that are in accordance with general anatomical knowledge (Mori et al., 1999; Conturo et al., 1999). The connectivity between voxels placed in grey matter is examined by means of a Monte-Carlo simulation, which allows particles to diffuse between voxels, with the probability for a "jump" in a particular direction being derived from the diffusion tensor in the voxel and the adjacent voxels. The anatomical connectivity between two voxels in grey matter is then defined in terms of the probability that a particle starting at one voxel will "diffuse" to the other.

For some functionally associated regions it has been shown that their spontaneous fluctuations in the BOLD signal are correlated (Biswal et al., 1995). This phenomenon is an example of functional connectivity as defined by Friston et al. (1993). Previously such regions have been defined by means of a conventional 
functional magnetic resonance (fMR) experiment and the correlation between signal fluctuations subsequently examined (Biswal et al., 1995; Lowe et al., 1998). There is, however, no explicit requirement to perform a standard fMR experiment to define these regions: anatomically defined regions of interest can equally well be employed. The practical advantage of this approach is that no stimulation paradigm is re quired, and hence all grey matter can in principle be investigated using this method. The measurement duration is also short: less than $10 \mathrm{~min}$ are usually sufficient. It has been assumed, though not conclusively proven, that the origin of spontaneous fluctuations in the BOLD signal is spontaneous bursts of electrical activity, which occur at low frequency in grey matter (Biswal et al., 1995). It has been shown that blood flow changes are associated with these bursts (Golanov et al., 1994), and it is a plausible assumption (Biswal et al., 1995) that these translate to changes in the BOLD signal intensity. It is known that the signal fluctuations arise from changes in BOLD contrast and are not the result of inflow effects (Biswal et al., 1997). In more conventional activation studies it has been shown that the degree of functional connectivity between regions can be modulated by such factors as the level of attention. For example, it has been demonstrated that the degree of connectivity between the motion-sensitive cortical area V5 and the posterior parietal cortex can be modified by the level of attention to visual motion (Büchel and Friston, 1998). By measuring spontaneous fluctuations it is hoped to examine a functional connectivity that is directly related to the underlying anatomy and not one modulated by the transient activity in the brain. In terms of the development of the brain this hypothesis is in accordance with Hebb's widely accepted theory (Hebb, 1949, p. 337) that cells which have a synchronized electrical activity will strengthen their synaptic connections (Frégnac, 1996). One example of this is spontaneous prenatal activity in the retina, which exerts a structuring influence on the development of the visual system (Meister et al., 1991). Further support is given by data suggesting that schizophrenia may well be caused by disconnection between cortical regions (Friston and Frith, 1995; Fletcher et al., 1999), combined with the discovery that the integrity of white matter tracts is compromised in this disease (Lim et al., 1999).

This study is to the best of the authors' knowledge the first to attempt to examine the relationship between functional and anatomical connectivity in a quantitative way. Previous investigations have generally attempted to relate changes in the diffusion tensor with functional deficits (Werring et al., 1998; Wieshmann et al., 1999; Klingberg et al., 2000). Although the data presented here are limited by not being acquired from a full 3-dimensional volume, a demonstration of principle is achieved that opens new avenues for future work in both the healthy and pathological brain.

\section{MATERIALS AND METHODS}

We investigated the anatomical and functional connectivity of cortical areas on adjacent gyri in 7 healthy volunteers who declared written informed consent. The experiments were generally approved by the ethics committee of Leipzig University. We restricted the study to cortical regions in close proximity to each other since long fibre tracts are more difficult to follow and are usually not confined to a single plane. In addition, it is known that in many cases the crowns of adjacent gyri are connected by U-shaped fibers.

The experiments were performed with a whole-body MRI system (Medspec 30/100, Bruker Medizintechnik, Ettlingen, Germany) operating at 3 Tesla (29.2 $\mathrm{mT} \mathrm{m}^{-1}$ max. gradient strength, $65 \mathrm{Ts}^{-1} \mathrm{~m}^{-1}$ slew rate, quadrature birdcage head resonator transmit/receive). For data evaluation the programming language IDL (Interactive Data Language, Research Systems I nc., CO) was used. The subjects were protected from acoustic noise by earplugs.

\section{BOLD I maging}

For fluctuation imaging we used a stimulus-free setup similar to that used by Biswal et al. (1995). In contrast to Biswal et al., however, we examined the correlation between cortical gyri in the same hemisphere rather than between symmetric areas in different hemispheres. Likewise, we did not perform a stimulus-induced activation experiment to identify the function of cortical regions. A time series of 1024 gradient-recalled blipped EPI images was acquired for a 5-mm axial slice $(64 \times 64$ matrix, $3 \mathrm{~mm}$ in-plane resolution, TR $=250 \mathrm{~ms}$, TE $=30 \mathrm{~ms}$, excitation with Ernst angle: $36^{\circ}$ ). The subjects were instructed to refrain from any voluntary movement during the approx. 4-min scan time. During the scan, acoustic and optical stimuli were absent apart from the noise produced by the scanner. The images were corrected for subject motion (Friston et al., 1996) and slightly smoothed. Then the signal time courses of all image pixels above a noise threshold were digitally low-pass $(<0.08 \mathrm{~Hz})$ frequency filtered (finite impulse response filter of order 40, see Press et al. (1992)) to remove signal fluctuations due to the respiratory and cardiac cycle (Biswal et al., 1995). The first 10 images were discarded to exclude the transition to steady state. Linear Pearson correlation coefficients (Pearson, 1896) between time courses were calculated for all possible pairs of pixels. In order to emphasize ubiquitious fluctuations that arise from spontaneous neuronal activity as opposed to those of limited duration due to other activity, each time course was split into four equal parts of about 


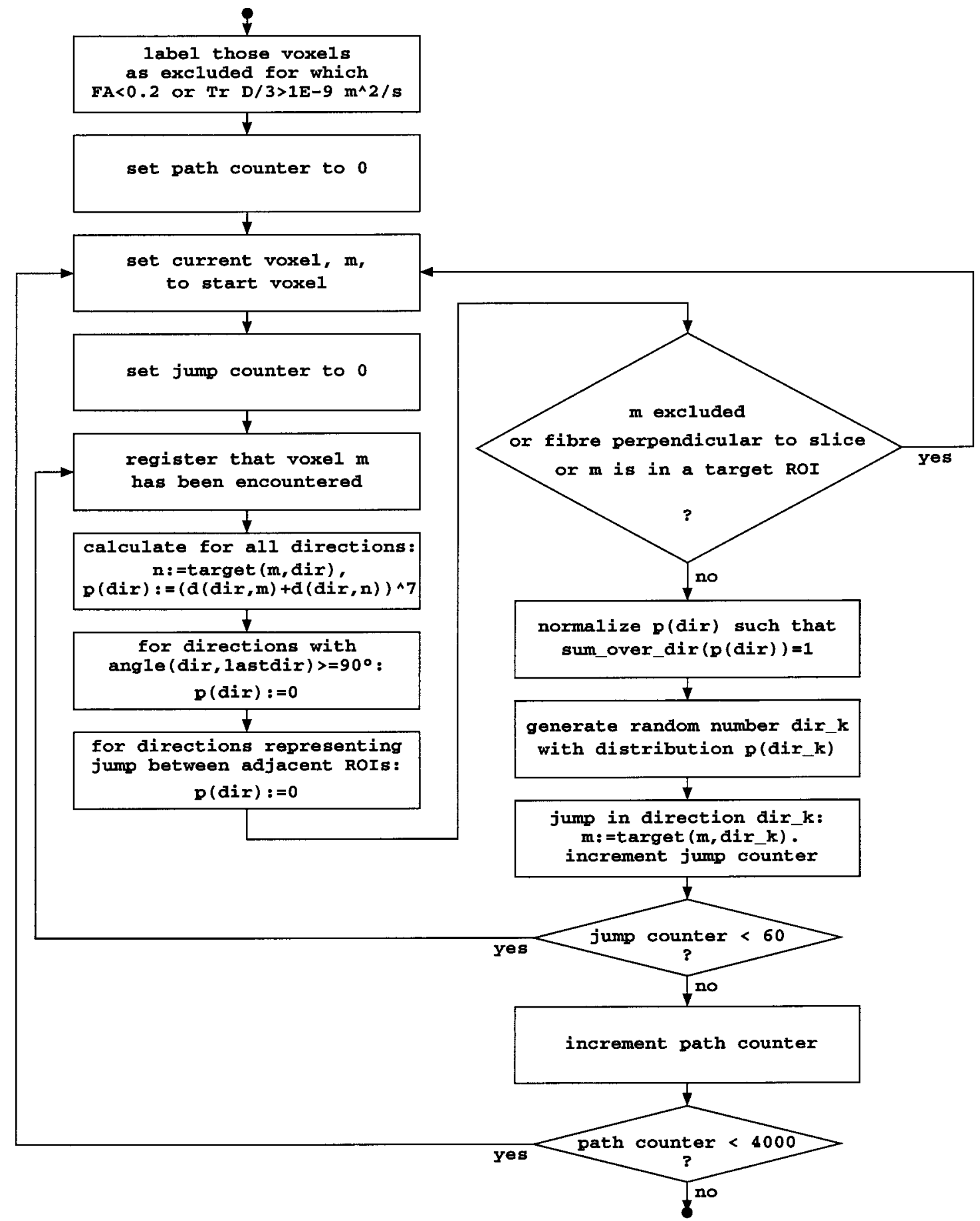

FIG. 1. Simplified flowchart of the algorithm for estimating anatomical connectivity from DTI data. The integer variables "dir" and "dir_k" specify one of the eight possible jump directions, " $\mathrm{m}$ " and " $\mathrm{n}$ " specify voxels. The sum over all possible directions is denoted by "sum_over_dir ()." The expression "target (m, dir)" is the target voxel of a jump from voxel " $\mathrm{m}$ " in direction "dir," and "d (dir, m) " is the diffusion coefficient defined in Eq. (3). The angle between the direction "dir" and the direction used for the preceding jump is denoted as "angle (dir, lastdir)." After normalization, "p (dir) "is the probability defined in Eq. (1). "Region of interest" is abbreviated as "ROI," and "a target ROI" means any region other than the region containing the start voxel. The condition "fiber perpendicular to slice" was evaluated by means of two diagonal elements of the diffusion tensor, see text. The trace of the tensor is denoted by "Tr D."

1-min duration. For each part the correlation coefficient between pixels was determined separately, and among the four coefficients the smallest one was selected for further processing. We expect to suppress "real task activation" to some degree by this procedure. 


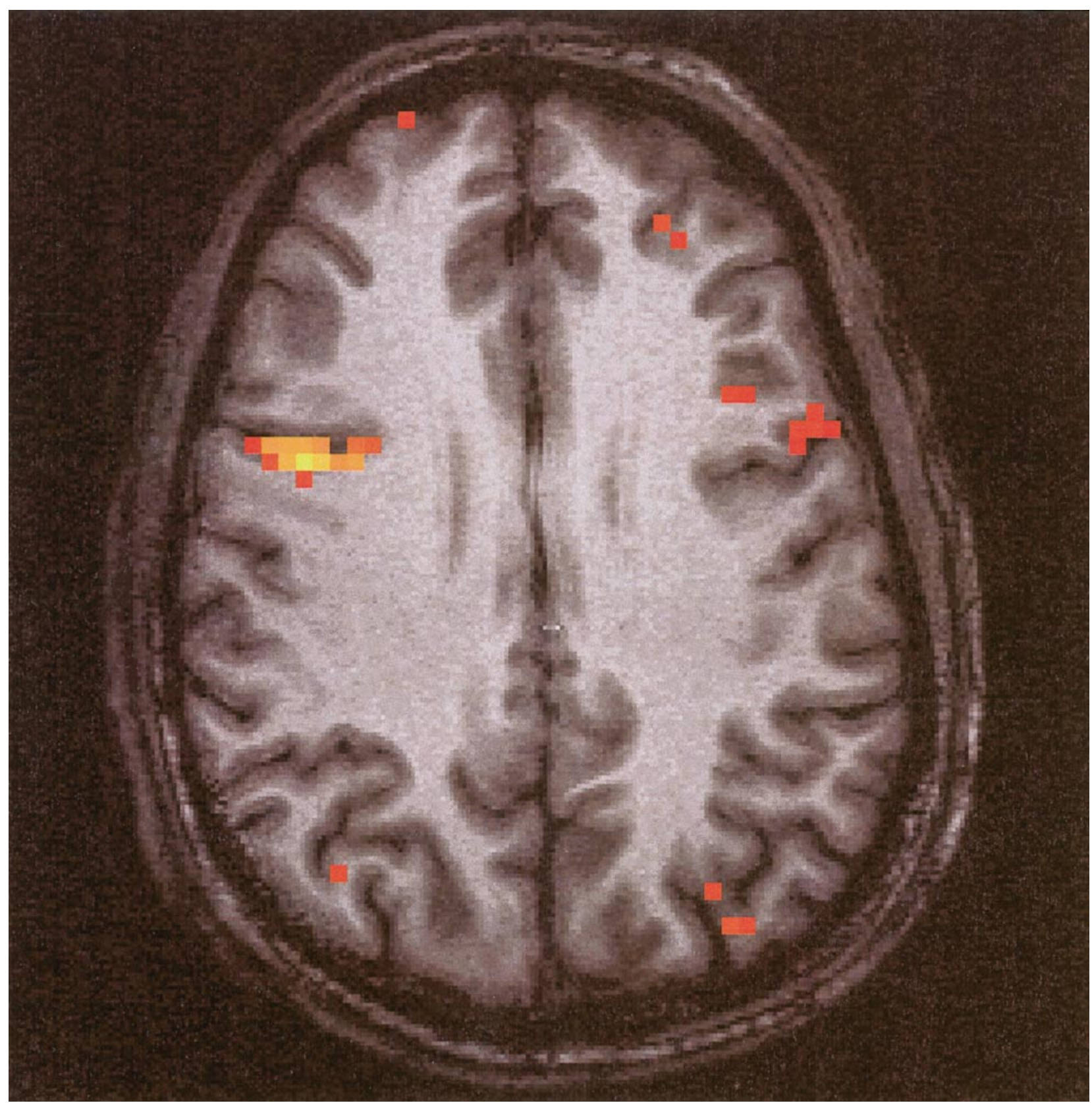

FIG. 2. Example for the pattern of correlation between BOLD signal time courses, overlaid on the corresponding $T_{1}$-weighted anatomical image. The color scale represents the magnitude of the correlation $\left(c_{f}\right)$ with the reference pixel which is shown in yellow (pixels with $c_{f}>0.4$ only).

\section{Diffusion Tensor Imaging}

Subsequent to the EPI scans, DTI was performed for the same slice in order to determine the anatomical connections between cortical areas. To achieve high spatial resolution we used a displaced U-FLARE (Norris et al., 1992) imaging sequence with spin-echo diffusion preparation (Stejskal and Tanner, 1965). We refrained from the use of EPI due to its lower spatial resolution and higher susceptibility to eddy current effects (Koch and Norris, 2000). In favor of high reso- lution, we accepted the lower SNR of U-FLARE and its inability to map a large number of slices in a short time. In order to reduce power deposition and effective echo time, we did not use dummy echoes for signal stabilization. I nstead, we determined a phase encoding order, which leads to a monotonous amplitude variation along the phase encode direction in k-space. This approach (TIPE, template interactive phase-encoding) was originally suggested for GRASE imaging (J ovicich and Norris, 1998). It requires the acquisition of a ref- 


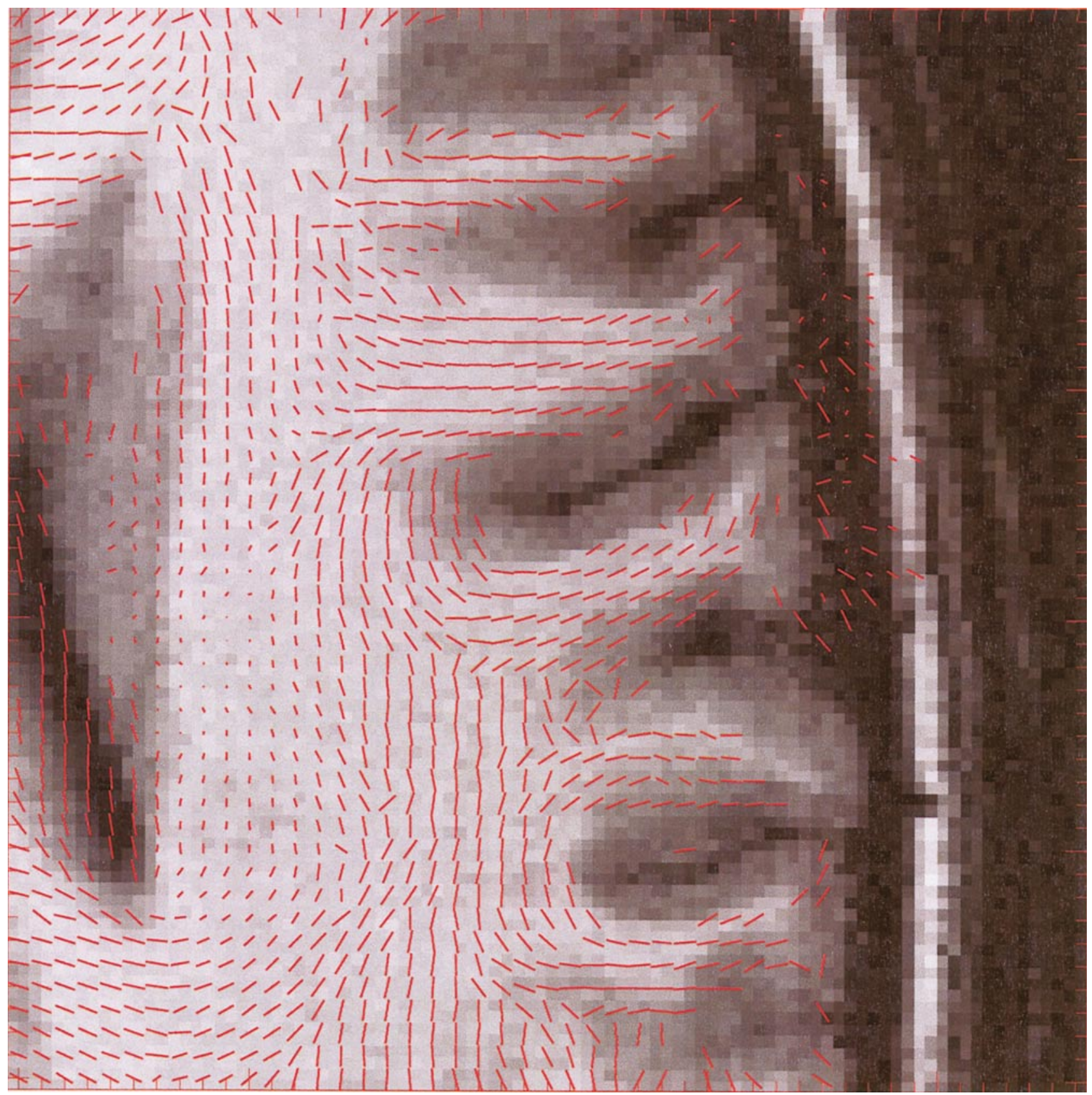

FIG. 3. Fiber orientation map calculated from the DTI data, overlaid on an anatomical $\mathrm{T}_{1}$-weighted image (transaxial slice, top is anterior). The straight lines represent the projection of the calculated fiber direction onto the image plane. In voxels with low anisotropy $(\mathrm{FA}<0.2)$ these lines are suppressed. The raw images were also masked to remove the background ghost signal in the phase encode direction (see text). Only a $6.75 \times 6.75-\mathrm{cm}$ part of the field-of-view is shown. The sulcus at the bottom is the postcentral sulcus.

erence scan without phase encoding gradients. The phase encoding gradients in the imaging sequence are ordered according to the echo amplitudes in the reference data. Since no even echo is present before the second refocusing pulse, one dummy cycle is still necessary. The phase encode direction was chosen along the volunteer's left-right direction to allow a minimum field-of-view (F OV). At $19.2 \times 14.4-\mathrm{cm}$ FOV, the $128 \times$ 96 data matrix corresponds to $1.5 \times 1.5-\mathrm{mm}$ nominal in-plane resolution (11.25- $\mathrm{mm}^{3}$ voxel volume). To reduce the number of RF pulses further, only 54 of 96 k-space lines were actually acquired. Zero-filling to the full $128 \times 96$ matrix before image reconstruction led to a slight decrease in spatial resolution. After reconstruction, the images were transformed to the $19.2 \times$ 19.2-cm format ( $128 \times 128$ pixels) by symmetric addition of zerolines. To ensure a sufficiently narrow point spread function (PSF), linear instead of center-out 
phase encoding was employed, starting six lines off the $\mathrm{k}$-space centre. The reduction in the number of RF pulses results in an overall SNR improvement because the refocusing angle can be increased without violation of power deposition limits, and because the U-FLARE echo train length is usually much larger than the optimum in terms of SNR. PSF broadening due to relaxation during the echo train did not exceed the width of a pixel. For the DTI experiment we used $\alpha \approx 120^{\circ}$ refocusing angle, interecho spacing $7.5 \mathrm{~ms}$, effective echo time $T E_{\text {eff }} \approx 140 \mathrm{~ms}$, and $T R=3 \mathrm{~s}$. We used diffusion gradients with 4 different amplitudes $(b=20$, $280,540,800 \mathrm{~s} \mathrm{~mm}^{-2}$ with approx. 15, 20, 30, 50 averages, respectively) and 7 directions $((1,1,0),(1,0,1)$, $(0,1,1),(1,-1,0),(-1,0,1),(0,-1,1),(1,1,1)$ in read-phase-slice coordinates). Separation and duration of the diffusion gradient pulses were $\Delta=40 \mathrm{~ms}$ and $\delta=$ $22 \mathrm{~ms}$, respectively. The acquisition of the tensor map took approximately $35 \mathrm{~min}$. Due to the high power deposition in U-FLARE, multislice imaging would have required a considerably longer scan time, or a reduction in the number of averages. A high quality tensor map of a single slice was preferred to multislice imaging with lower effect-to-noise ratio.

\section{Estimating Anatomical Connectivity from DTI Data}

For the assessment of anatomical connectivity between brain regions we implemented a Monte-Carlo type algorithm. It is based on the idea of a particle that performs a macroscopic random walk through the set of voxels. However, the probability for a jump in a given direction was chosen to depend monotonously on the diffusion coefficient along the jump direction in the start and target voxel of the jump. The particle then moves with a higher probability along the fiber direction than perpendicular to it. In a number of such random walks starting in a region " $A$," it is counted how often some other region " $B$ " was reached. This yields a relative measure of the anatomical connectivity between the regions " $A$ " and "B." For each elementary jump, the probability for a jump from the start voxel, $m$, to the neighboring voxel $n$ in the imaging slice was set to

$$
\mathrm{p}(\mathrm{m} \rightarrow \mathrm{n})=\frac{\left[\mathrm{d}\left(\mathbf{r}_{\mathrm{mn}}, \mathrm{m}\right)+\mathrm{d}\left(\mathbf{r}_{\mathrm{mn}}, \mathrm{n}\right)\right]^{\mathrm{a}}}{\sum_{\mathrm{n}^{\prime}=1}^{8}\left[\mathrm{~d}\left(\mathbf{r}_{\mathrm{mn}}, \mathrm{m}\right)+\mathrm{d}\left(\mathbf{r}_{\mathrm{mn}}, \mathrm{n}^{\prime}\right)\right]^{\mathrm{a}}},
$$

with $\mathrm{a}=7$, where $\mathrm{d}\left(\mathbf{r}_{\mathrm{mn}}, \mathrm{m}\right)$ is the "diffusion coefficient" in voxel $m$ along the line connecting the centers of $m$ and $n$. The definition of this coefficient is based on Einstein's equation (Einstein, 1905) for the r.m.s. displacement of a freely diffusing particle after a time $t$, which states $\left\langle\mathbf{s}_{\mathrm{x}}^{2}(\mathrm{t})\right\rangle=2 \mathrm{tD}$ for the $\mathrm{x}$ component of the displacement $\mathbf{s}(\mathrm{t})$, where $\mathrm{D}$ is the diffusion coefficient and $\langle\ldots\rangle$ represents an ensemble average. This equation can be generalized to an expression for the r.m.s. displacement along an arbitrary unit vector $\check{\text { r, }}$

$$
\left\langle(\mathbf{s}(\mathrm{t}) \cdot \check{\mathbf{r}})^{2}\right\rangle=2 \mathrm{t} \check{\mathbf{r}} \cdot \mathrm{D} \check{\mathbf{r}}
$$

for diffusion in a not necessarily isotropic medium which is characterized by the diffusion tensor $D$. Hence, the "diffusion coefficient $d(r, m)$ in voxel $m$ along $\mathbf{r}$ " can be defined by

$$
d(\mathbf{r}, m)=\check{\mathbf{r}} \cdot D(m) \check{\mathbf{r}}
$$

where $D(m)$ is the diffusion tensor in voxel $m$, and $\check{\mathbf{r}}=$ $\mathbf{r} /|\mathbf{r}|$. This definition was used in $\mathrm{Eq}$. (1). The probability defined in Eq. (1) satisfies $\sum_{n=1}^{8} p(m \rightarrow n)=1$. The exponent a was introduced to make the probability distribution sufficiently narrow. Let us consider a homogeneous sample for simplicity, where $\mathrm{d}\left(\mathbf{r}_{\mathrm{mn}}, \mathrm{m}\right)=$ $\mathrm{d}\left(\mathbf{r}_{\mathrm{mn}}, \mathrm{n}\right)=: \mathrm{d}\left(\mathbf{r}_{\mathrm{mn}}\right)$. Then Eq. (1) implies

$$
\frac{p^{a=1}(m \rightarrow n)}{p^{a=1}\left(m \rightarrow n^{\prime}\right)}=\frac{d\left(r_{m n}\right)}{d\left(r_{m n^{\prime}}\right)} \ll\left(\frac{d\left(r_{m n}\right)}{d\left(r_{m n^{\prime}}\right)}\right)^{7}=\frac{p^{a=7}(m \rightarrow n)}{p^{a=7}\left(m \rightarrow n^{\prime}\right)}
$$

for the jump probabilities to the voxels $n$ and $n^{\prime}$, given that $\mathrm{d}\left(\mathbf{r}_{\mathrm{mn}}\right)<\mathrm{d}\left(\mathbf{r}_{\mathrm{mn}}\right)$. The chosen value of a was such that most particle paths followed the fibers on the DTI fiber orientation map. To ensure a minimum smoothness of the particle path, the particle was only allowed to jump in a direction that deviated by $<90^{\circ}$ from the preceding jump direction, i.e., only the 3 "forward" jump directions were possible. We excluded (i) voxels where the fractional anisotropy index FA (Basser and Pierpaoli, 1996) was less than $F A=0.2$ and (ii) voxels where trace(D)/3>10 $10^{-9} \mathrm{~m}^{2} \mathrm{~s}^{-1}$. These threshold values varied slightly between subjects and were adjusted to suppress grey matter (low FA) and CSF (high trace(D) but possibly high FA due to flow) as accurately as possible. Before tensor calculation, a brain mask was applied to the U-FLARE images. For each jump, a pseudo-random integer, n, between 0 and 7 was generated and used to select the jump direction. The transformation method (Press et al., 1992) was used to achieve that these numbers were distributed according to Eq. (1). The particle path was terminated when an excluded voxel was reached or when 60 jumps had been performed. This maximum number of jumps was well above the minimum number of jumps required for the particle to travel from a gyrus crown to a neighboring crown. The path was also terminated if the sum of the in-plane diagonal tensor elements was less than $10^{-9}$ $\mathrm{m}^{2} \mathrm{~s}^{-1}$. This rule terminates the path where the fibre direction is perpendicular to the slice, allowing for paths to leave the image plane. The frequency with 
which each voxel was encountered (as a result of any particle jump during a path or at its terminating point) during 4000 paths was recorded and divided by the maximum over all pixels in the slice. Increasing the number of experiments beyond 4000 did not alter the results significantly. Figure 1 shows a flowchart of the program which was written in the programming language $C$.

Comparing the simulation results with functional connectivity is not straightforward since DTI provides reliable information on fiber orientation only for white matter (WM), whereas BOLD fluctuations occur predominantly in grey matter (GM). It is difficult to decide which of the voxels in superficial WM contains the extracortical connections of a given GM voxel. The situation may be further complicated because EPI images are prone to distortions ( ohnson and Hutchison, 1985), in contrast to U-FLARE images. We therefore manually defined regions of interest in the $64 \times 64$ matrix on the crowns of selected gyri on the convex surface of the brain, containing subcortical WM voxels that could serve as starting pixels in the simulation. A region consisted of typically ten pixels of the $64 \times 64$ matrix. Three of the seven volunteers in the study did not clearly exhibit U-shaped fibers between adjacent gyri in the DTI maps and were excluded from the simulation. This criterion excludes volunteers where we might have failed to map such fiber tracts due to inadequate slice position. The Monte-Carlo simulation was performed on the $128 \times 128$ DTI map and started from the voxels that belonged to one of the regions of interest. As some regions of interest were directly adjacent to another region, direct jumps between adjacent regions were explicitly excluded. It was further verified that the correlation coefficient was below 0.2 for any two neighboring voxels which belonged to different regions. The particle paths were terminated if a region of interest other than the starting region was reached. Finally, a value of anatomical $\left(c_{d}\right)$ and functional $\left(c_{f}\right)$ connectivity was assigned to every ordered pair of regions by taking the maximum of $c_{d}$ and $c_{f}$ in all pixels in both regions. The matrix of the $c_{d}$ values is not necessarily symmetric, in contrast to the $c_{f}$ matrix. As it is not obvious how these matrices should be compared, the $c_{d}$ values for both possible choices of the starting region were computed and displayed.

\section{RESULTS}

All seven subjects in the study (including those which were excluded from the DTI evaluation) showed high correlations between various cortical areas. In contrast, most of the white matter voxels exhibited very low correlation with other voxels. Many grey matter voxels showed high correlation with voxels approximately at the corresponding location in the contralateral hemisphere. This observation is in agreement with the results of Biswal et al. (1995). An example of a correlation map is shown in Fig. 2. The typical signal variation during the time course of $4 \mathrm{~min}$ is of the order of $3 \%$. This is compatible with the variation of 0.5 to $1 \%$ observed by Biswal et al. at $1.5 \mathrm{~T}$ main field strength when the difference in $B_{0}$ is taken into account. Translation due to subject motion during the BOLD scan was bel ow 0.2 voxels. An example of a fiber orientation map calculated from the acquired DTI data is shown in Fig. 3. As an example of the result of the simulation, Fig. 4 demonstrates that the most frequently encountered voxels correspond to those that constitute a fiber tract in the fiber orientation map according to subjective perception. For each pair of regions on adjacent gyri in the four subjects evaluated, Fig. 5 shows the correlation coefficients and the simulation results based on the DTI experiment. The anatomical connectivity is given for both possible choices of the start region. For some region pairs the difference between the simulation results with start and target region interchanged is very large. There is also a considerable variation in anatomical and functional connectivity between adjacent gyri, which is not due to interindividual differences alone as there is also a substantial variation for each subject (not shown).

In Fig. 5, high $c_{d}$ values seem not to occur in combination with low $c_{f}$ values. Moreover, a positive correlation between $c_{f}$ and $c_{d}$ was evident when considering region pairs around the central sulcus only.

\section{DISCUSSION}

The absolute number of axons connecting two cortical regions can hardly be calculated from DTI because the diffusion tensor depends on density and diameter of axons, degree of axon myelination, and variance in axon direction. However, the particle jump simulation provides a relative estimate of the number of connecting axons, as the following argument shows. The simulation result for paths from a region " $A$ " to a region " $B$," $C_{d}(A \rightarrow B)$, is high if a fiber tract exists between " $A$ " and "B." It is reduced if a large number of possibilities is encountered on the way between " $A$ " and " $B$ " to divert from the track leading to "B." A diverging fiber path means that the population of axons in the first voxel(s) of the tract can be divided into two groups with different targets. Let us assume that $n_{i}$ axons leave or enter a region " $A_{i}$," $n_{j}$ axons leave or enter some other region " $A_{j}$," and $k_{i j}$ axons connect these regions. We can then interpret $c_{d}\left(A_{i} \rightarrow A_{j}\right)$ as an estimate of $k_{i j} / n_{i}$, and $c_{d}\left(A_{j} \rightarrow A_{i}\right)$ as an estimate of $k_{i j} / n_{j}$. Hence, $c_{d}\left(A_{i} \rightarrow A_{j}\right)$ estimates the fraction of axons originating in " $A_{i}$ " that represent a link with " $A_{j}$." This justifies the term "anatomical connectivity" for $c_{d}$. The case $c_{d}(A \rightarrow B) \neq$ $C_{d}(B \rightarrow A)$ reflects that two brain regions may maintain connections to a different number of areas (Felleman and Van Essen, 1991). 

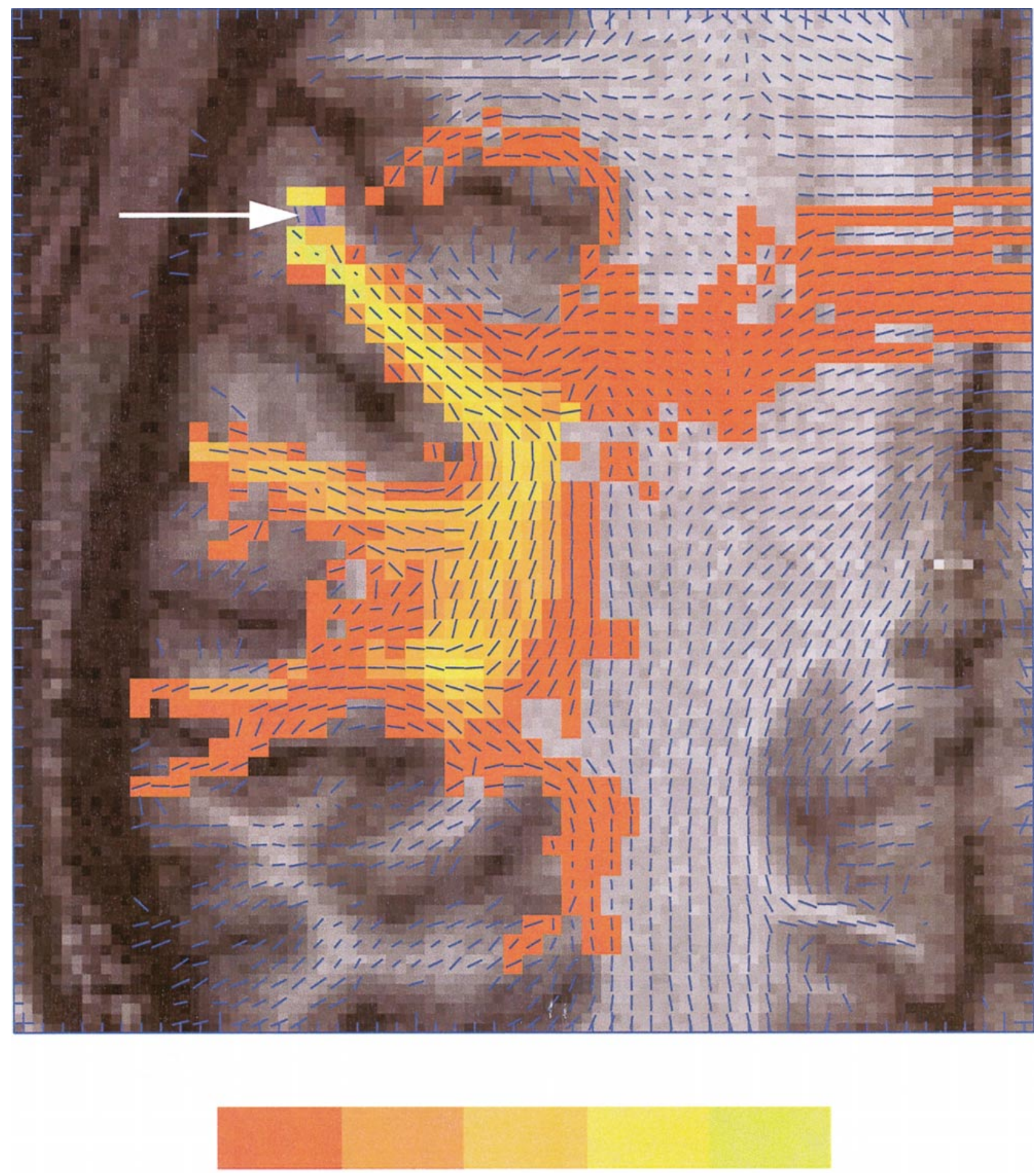

0.05

0.1

0.2

0.4

FIG. 4. Example for the result of the particle-jump algorithm. The start pixel is shown in blue and marked with an arrow. The $c_{d}$ values for individual pixels are indicated by colors: red (yellow) voxels were crossed by a low (high) number of particle paths. The colors for five selected $c_{d}$ values are shown in the col or scale. Uncol ored pixels were never reached by the particle. Fiber directions as described in the legend of Fig. 3 are shown in blue. 


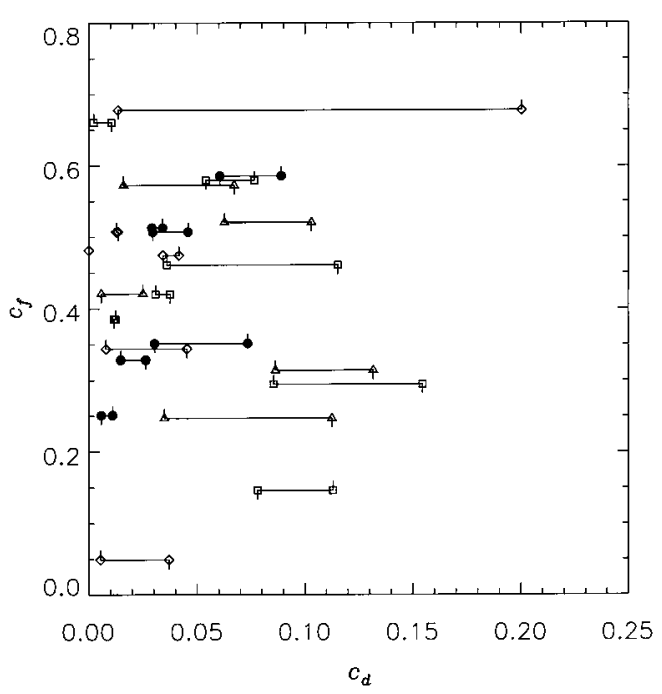

FIG. 5. Correlation coefficient $c_{f}$ between regions on adjacent gyri versus the corresponding anatomical connectivity measure $c_{d}$. Each region pair is represented by two symbols: since in the simulation the start pixel can be set in either of the two regions, two $c_{d}$ values per region pair are shown, connected by a horizontal line. Symbols with vertical lines pointing downwards represent paths where the starting region is more anterior, symbols with lines pointing upward represent paths where the starting region is more posterior. Identified location of evaluated gyrus pairs: inferior frontal and precentral gyrus (diamond), precentral and postcentral gyrus (filled circle), and postcentral and supramarginal gyrus (triangle). All other gyrus pairs are shown with squares. The plot comprises the data from left and right hemispheres of the four evaluated subjects (the number of regions differs between subjects).

If DTI-visible fiber connections are the basis for the observed correlations then the $c_{f}$ distribution should depend on the $c_{d}$ value. This holds even if we are not able to register all existing connections. The data presented in Fig. 5 provide some support for such a dependence. There are a number of reasons why a straightforward correlation between anatomical and functional connectivity may not be expected. Observed signal correlations between two cortical regions could be mediated by indirect anatomical connections. In this case the BOLD signal times courses may be highly correlated, although no connecting fiber is present in the DTI fiber map. Such cases are likely to exist since an interhemispheric correlation of BOLD signal fluctuations has been observed in cortical regions that have only few direct commissural connections, such as the left and right hand representation in the primary motor cortex (Biswal et al., 1995) and the left and right primary visual cortex (Lowe et al., 1998). The occurrence of high $c_{f}$ values at low $c_{d}$ values in Fig. 5 is consistent with these observations. Furthermore, we cannot detect intracortical connections or genuine $U$ fibers (Miodoński, 1974) in superficial WM. Our protocol also misses out fibers that are not contained in the image slice. On the other hand, fiber bundles in DTI fiber maps do not necessarily represent a continuous fiber tract. As one proceeds along a fiber bundle, the axons may gradually be replaced by other axons joining the bundle, which is invisible in the DTI map. This could explain the group of points in Fig. 5 with $\mathrm{C}_{\mathrm{f}}<0.4$ and $0.075<c_{d}<0.16$. The observation in Fig. 5 that only the region pairs around the central sulcus show a clear correlation between $c_{d}$ and $c_{f}$ might be explained by anatomical arguments. Cytoarchitecture, gross function, and probably connection pattern of the cortical areas on both sides of the central sulcus are more or less the same over the length of the sulcus, in contrast to the pre or postcentral sulcus (Brodmann, 1909, p. 153). This implies a reduced variance contribution from a varying slice position.

Conventional fiber tracking algorithms (Conturo et al., 1999; Mori et al., 1999; J ones et al., 1999) usually generate a trajectory by following the direction corresponding to the largest eigenvalue of the diffusion tensor. However, this approach does not provide a quantitative measure of anatomical connectivity. In addition, it does not discriminate between voxels with the same principal directions but different degrees of anisotropy, and isotropic regions cannot be traversed. The algorithm presented here is substantially different. It provides a quantitative measure of anatomical connection strength, and it reflects the statistical nature of the information obtained with DTI. It can deal with fiber bifurcations and with isotropic voxels. Because the full tensor information is used, the degree of anisotropy is taken into account. The algorithm can easily be adapted to 3-dimensional DTI data (Koch et al., 2001). In order to incorporate data from 3-dimensional q-space imaging (Tuch et al., 1999; Wedeen et al., 2000), the calculation of jump probabilities could be based on the measured probability distribution of molecular displacements rather than on the diffusion tensor. In the relatively isotropic region at a fiber crossing, the particle paths would then follow the directions of the crossing fibers with a higher probability.

In this article we presented a new approach for extracting a quantitative measure of anatomical connectivity from DTI data. A comparison of anatomical and functional connectivity between regions of interest on adjacent cortical gyri revealed that low functional connectivity rarely occurs in combination with high anatomical connectivity. I n contrast, high functional and low anatomical connectivity do occur in combination. This is interpreted as an indication of BOLD signal correlation mediated by undetected or indirect connections. Using a 3-dimensional DTI data set, the presented approach is expected to shed further light on the anatomical foundations of functional connectivity.

\section{ACKNOWLEDGMENTS}

M.A.K. enjoyed partial financial support from the BIOMED II scheme of the European Union project BMH4-96-0861. Echo order- 
ing for TIPE phase encoding was supplied by J . J ovicich and adapted to linear encoding by $\mathrm{V}$. Nebe.

\section{REFERENCES}

Basser, P. J ., Mattiello, J ., and Le Bihan, D. 1994a. MR diffusion tensor spectroscopy and imaging. Biophys. J . 66: 259-267.

Basser, P. J ., Mattiello, J ., and LeBihan, D. 1994b. Estimation of the effective self-diffusion tensor from the NMR spin echo. J. Magn. Reson. B 103: 247-254.

Basser, P. J ., and Pierpaoli, C. 1996. Microstructural and physiological features of tissues elucidated by quantitative-diffusion-tensor MRI. J . Magn. Reson. B 111: 209-219.

Biswal, B. B., Van Kylen, J., and Hyde, J. S. 1997. Simultaneous assessment of flow and BOLD signals in resting-state functional connectivity maps. NMR Biomed. 10: 165-170.

Biswal, B. B., Yetkin, F. Z., Haughton, V. M., and Hyde, J . S. 1995. Functional connectivity in the motor cortex of resting human brain using echo-planar MRI. Magn. Reson. Med. 34: 537-541.

Brodmann, K. 1909. Vergleichende Lokalisationsl ehre der Großhirnrinde, in ihren Prinzipien dargestelt auf Grund des Zellenbaues, 1st ed., reprint 1985. J . A. Barth, Leipzig.

Büchel, C. and Friston, K. J . 1998. Dynamic changes in effective connectivity characterized by variable parameter regression and Kalman filtering. Hum. Brain Mapp. 6: 403- 408.

Conturo, T. E., Lori, N. F., Cull, T. S., Akbudak, E., Snyder, A. Z., Shimony, J. S., McKinstry, R. C., Burton, H., and Raichle, M. E. 1999. Tracking neuronal fiber pathways in theliving human brain. Proc. Natl. Acad. Sci. USA 96: 10422-10427.

Einstein, A. 1905. Über die von der molekularkinetischen Theorie der Wärme geforderte Bewegung von in ruhenden Flüssigkeiten suspendierten Teilchen. Annalen Physik Chemie (4. Folge) 17: 549-560.

Felleman, D. J . and Van Essen, D. C. 1991. Distributed hierarchical processing in the primate cerebral cortex. Cerebral Cortex 1: 1- 47.

Fletcher, P., McKenna, P. J ., Friston, K. J ., Frith, C. D., and Dolan, R. J. 1999. Abnormal cingulate modulation of fronto-temporal connectivity in schizophrenia. Neuroimage 9: 337-342.

Frégnac, Y. 1996. Dynamics of functional connectivity in visual cortical networks: An overview. J . Physiology (Paris) 90: 113-139.

Friston, K. J . and Frith, C. D. 1995. Schizophrenia: A disconnection syndrome? Clin. Neurosci. 3: 89-97.

Friston, K. J ., Frith, C. D., Liddle, P. F., and Frackowiak, R. S. J . 1993. Functional connectivity: The principal-component analysis of large (PET) data sets. J . Cereb. Blood Flow Metab. 13: 5-14.

Friston, K. J ., Williams, S., Howard, R., Frackowiak, R. S. J ., and Turner, R. 1996. Movement-related effects in fMRI time-series. Magn. Reson. Med. 35: 346-355.

Golanov, E. V., Yamamoto, S., and Reis, D. J . 1994. Spontaneous waves of cerebral blood flow associated with a pattern of electrocortical activity. Am. J . Physiol. 266: R204-R214.

Hebb, D. O. 1949. The Organization of Behavior. Wiley, New York.

J ohnson, G., and Hutchison, J . M. S. 1985. The limitations of NMR recalled-echo imaging techniques. J . Magn. Reson. 63: 14-30.

J ones, D. K., Simmons, A., Williams, S. C. R., and Horsfield, M. A. 1999. Non-invasive assessment of axonal fiber connectivity in the human brain via diffusion tensor MRI. Magn. Reson. Med. 42: 37- 41.

J ovicich, J ., and Norris, D. G. 1998. GRASE Imaging at 3 Tesla with template interactive phase-encoding. Magn. Reson. Med. 39: 970979.

Klingberg, T., Hedehus, M., Temple, E., Salz, T., Gabrieli, J . D. E., Moseley, M. E., and Poldrack, R. A. 2000. Microstructure of tem- poro-parietal white matter as a basis for reading ability: Evidence from diffusion tensor magnetic resonance imaging. Neuron 25: 493-500.

Koch, M., Glauche, V., Finsterbusch, J ., Nolte, U., Frahm, J ., and Büchel, C. 2001. Estimation of anatomical connectivity from diffusion tensor data. Neuroimage 13: S176.

Koch, M. and Norris, D. G. 2000. An assessment of eddy current sensitivity and correction in single-shot diffusion-weighted imaging. Phys. Med. Biol. 45: 3821-3832.

Lim, K. O., Hedehus, M., Moseley, M., de Crespigny, A., Sullivan, E. V., and Pfefferbaum, A. 1999. Compromised white matter tract integrity in schizophrenia inferred from diffusion tensor imaging. Arch. Gen. Psychiatry 56: 367-374.

Lowe, M. J., Mock, B. J ., and Sorenson, J. A. 1998. Functional connectivity in single and multislice echoplanar imaging using resting-state fluctuations. Neuroimage 7: 119-132.

Meister, M., Wong, R. O. L., Baylor, D. A., and Shatz, C. J . 1991. Synchronous bursts of action potentials in ganglion cells of the developing mammalian retina. Science 252: 939-943.

Miodoński, A. 1974. The angioarchitectonics and cytoarchitectonics (impregnation modo Golgi-Cox) structure of the fissural frontal neocortex in dog. Folia Biol. (Kraków) 22: 237-279.

Mori, S., Crain, B. J ., Chacko, V. P., and van Zijl, P. C. M. 1999. Three-dimensional tracking of axonal projections in the brain by magnetic resonance imaging. Ann. Neurol. 45: 265-269.

Norris, D. G., Börnert, P., Reese, T., and Leibfritz, D. 1992. On the application of ultra-fast RARE experiments. Magn. Reson. Med. 27: 142-164.

Ogawa, S., Lee, T. M., Kay, A. R., and Tank, D. W. 1990a. Brain magnetic resonance imaging with contrast dependent on blood oxygenation. Proc. Natl. Acad. Sci. USA 87: 9868-9872.

Ogawa, S., Lee, T. M., Nayak, A. S., and Glynn, P. 1990b. Oxygenation-sensitive contrast in magnetic resonance image of rodent brain at high magnetic fields. Magn. Reson. Med. 14: 68-78.

Pearson, K. 1896. Mathematical contributions to the theory of evolution, III. Regression, heredity, and panmixia. Phil. Trans. R. Soc. Lond. A 187: 253-318.

Press, W. H., Teukolsky, S. A., Vetterling, W. T., and Flannery, B. P. 1992. Numerical Recipes in C. The Art of Scientific Computing. Cambridge Univ. Press, Cambridge, 2nd ed.

Stejskal, E. O., and Tanner, J. E. 1965. Spin diffusion measurements: Spin echoes in the presence of a time-dependent field gradient. J . Chem. Phys. 42: 288-292.

Tuch, D. S., Weisskoff, R. M., Belliveau, J. W., and Wedeen, V. J . 1999. High angular resolution diffusion imaging of the human brain. In Proceedings, ISMRM, 7th Annual Meeting, p. 321. Philadelphia, PA.

Wedeen, V. J ., Reese, T. G., Tuch, D. S., Weigel, M. R., Dou, J .-G., Weiskoff, R. M., and Chessler, D. 2000. Mapping fiber orientation spectra in cerebral white matter with Fourier-transform diffusion MRI. In Proceedings, ISMRM, 8th Annual Meeting, p. 82. Denver, CO.

Werring, D. J ., Clark, C. A., Barker, G. J ., Miller, D. H., Parker, G. J . M., Brammer, M. J ., Bullmore, E. T., Giampietro, V. P., and Thompson, A. J . 1998. The structural and functional mechanisms of motor recovery: Complementary use of diffusion tensor and functional magnetic resonance imaging in a traumatic injury of the internal capsule. J. Neurol. Neurosurg. Psychiatry 65: 863869.

Wieshmann, U. C., Clark, C. A., Symms, M. R., Franconi, F., and Barker, G. J . Shorvon, S. D. 1999. Anisotropy of water diffusion in corona radiata and cerebral peduncle in patients with hemiparesis. Neuroimage 10: 225-230. 